\title{
Demonstrator System for the Phase-I Upgrade of the Trigger Readout Electronics of the ATLAS Liquid Argon Calorimeters
}

\author{
Kai Chen \\ Physics Department, Brookhaven National Laboratory \\ Upton, NY 11973, USA \\ On behalf of the ATLAS Liquid Argon Calorimeter Group
}

\begin{abstract}
The trigger readout electronics of the ATLAS Liquid Argon (LAr) Calorimeters will be improved for the PhaseI luminosity upgrade of the $\mathrm{LHC}$, to enhance the trigger feature extraction. Signals with higher spatial granularity will be digitized and processed by newly developed front-end and back-end electronics. In order to evaluate the technical and performance aspects, a demonstrator system has been set up, and many off-detector tests have been done. Analog signal parameters including the noise and cross-talk have been measured. Digital signal treatment, and high-speed data transmission have been tested and verified. After a series of tests, the demonstrator system has been installed on the ATLAS detector for the LHC Run 2.
\end{abstract}

Index Terms-ATLAS, Liquid Argon Calorimeters, Trigger Readout Electronics.

\section{INTRODUCTION}

$\mathbf{T}$ HE second long shutdown of LHC is scheduled in 20182019. For LAr Calorimeters of the ATLAS detector[1], the Phase-I upgrade of the trigger readout electronics will be installed. The objective of this upgrade is to provide highergranularity, higher-resolution and longitudinal shower information from the calorimeter to the Level-1 trigger processors. The improved read-out of the trigger signals will process 34000 so-called super-cells at every LHC bunch-crossing at a frequency of $40 \mathrm{MHz}$.

The existing calorimeter trigger information is based on the concept of a "Trigger Tower" that sums the energy deposition across all of the four longitudinal layers of the calorimeter in an area of $\Delta \eta \times \Delta \varphi=0.1 \times 0.1$, shown as the Figure 1 . The new scheme with finer granularity is based on super-cells. The transverse energy in each layer is retained. As shown in Figure 2, it provides information for each calorimeter layer for the full $\eta$ range of calorimeters, as well as finer segmentation $(\Delta \eta \times \Delta \varphi=0.025 \times 0.1)$ in the front and middle layers of the EM barrel and endcap for $|\eta| \leq 2.5[3]$.

As the LHC luminosity increases above the LHC design value, the improved calorimeter trigger electronics will allow ATLAS to deploy more sophisticated algorithms, enhancing the ability to measure the properties of the newly discovered Higgs boson and the potential for discovering physics beyond the Standard Model.

A demonstrator system has been set up to evaluate the technical and performance aspects of the LAr trigger readout

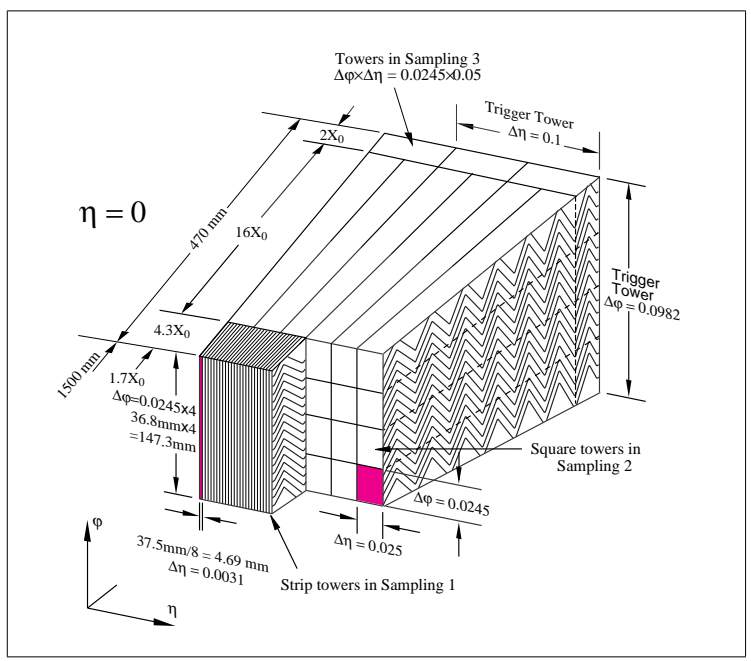

Fig. 1: Sketch of the accordion structure of the existing EM calorimeter[2]. Three longitudinal layers (front, middle and back), are shown in the figure, while the pre-sampler layer in front of the calorimeter is not shown.

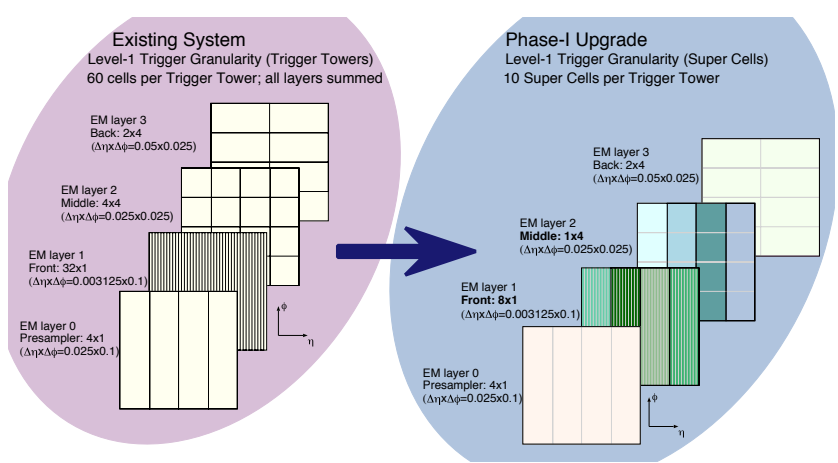

Fig. 2: Geometrical representation in $\eta, \phi$ space of an EM Trigger Tower in the current system, where the transverse energy in all four layers are summed (left) and of the supercells proposed for the Phase-I upgrade, where the transverse energy in each layer is retained in addition to the finer granularity in the front and middle layers (right). Each big square here represents an area of size $\Delta \eta \times \Delta \phi=0.1 \times 0.1$.[3]

upgrade. The analog trigger system will remain unchanged 
during LHC Run 2 and Run 3. The Phase-I system will use radiation qualified parts, mainly ASICs, while for the demonstrator which has been installed, some commercial offthe-shelf chips, such as the analog to digital converter, are used.

\section{The Demonstrator System}

\section{A. The Phase-I Upgrade Schematic}

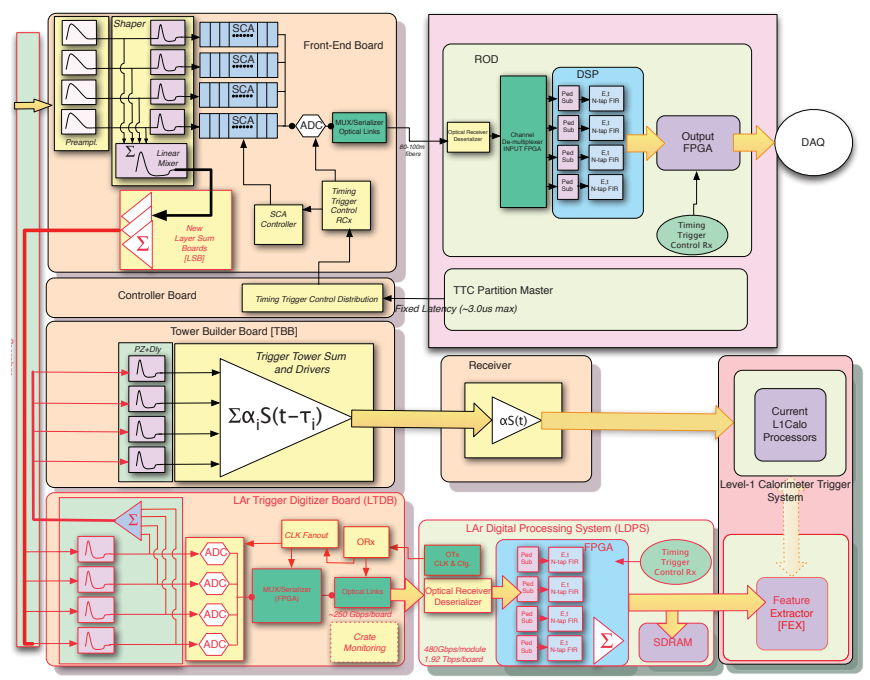

Fig. 3: Schematic block diagram of the upgraded LAr trigger readout architecture[3]

The architecture of the Phase-I upgrade trigger electronics is depicted in Figure 3, with the upgraded and new components outlined in red. New LAr Trigger Digitizer Boards (LTDB) will be installed in the available spare slots of the Front-End crates.

For the existing readout system, the front-end boards will be kept unchanged. The upgraded readout system will use the new layer sum boards, and the new baseplanes. These updated components will allow the LTDBs to digitize information with better granularity in the front and middle layers of the EM calorimeters.

\section{B. Boards of the Demonstrator System}

The LTDB demonstrator board will digitize up to 320 supercell signals with 12-bit ADCs running at 40 mega-samples per second, then stream the data out of the detector with serializers and fiber optical links. There are 8 channels in each ADC, and they will share one optical link. A customized protocol using 8B10B coding is used for the data transmission, with a line rate of the data links at $4.8 \mathrm{Gbps}$. The LTDB also builds the analog sums, and sends them to the current trigger system, through the new baseplane. Figure 4 shows the two different LTDB demonstrator boards. One of them uses digital mother board and analog mezzanine, the other one uses analog motherboard and digital mezzanine.

Figure 5 shows the ATCA test Board for Baseline Acquisition (ABBA), which works as the LAr Digital Processing Board. It receives the data from the LTDB demonstrator through the

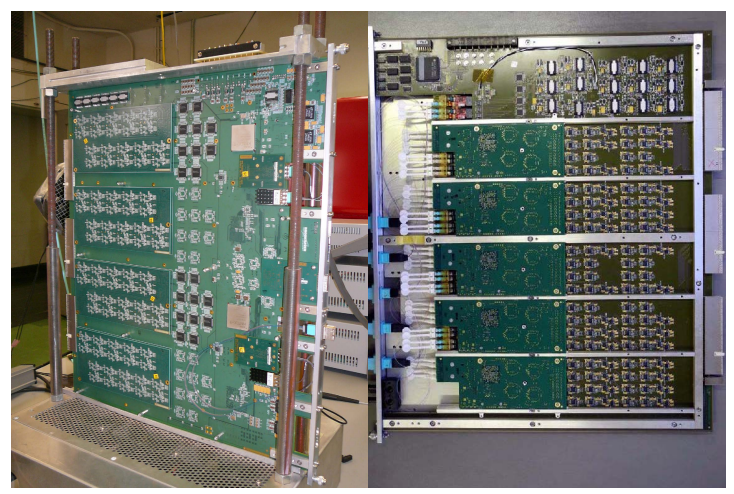

Fig. 4: Two LTDB demonstrator boards

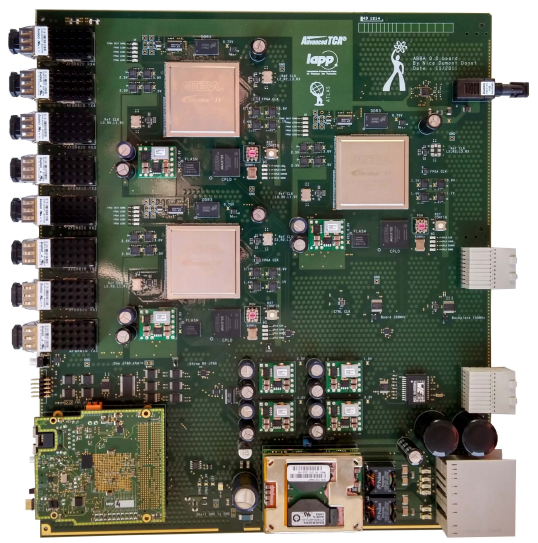

Fig. 5: The ABBA board

4.8 Gbps optical links, and reconstructs energy with the digital filter algorithm, then sends the data to a PC. The PC is used to control and configure the ABBA board using trigger and data acquisition system, and the LAr online system. The interface between them is 10-gigabit Ethernet.

\section{Preliminary Test Results at LAr Electronic Maintenance Facility}

Before the installation, extensive tests have been done at the LAr Electronic Maintenance Facility at CERN.

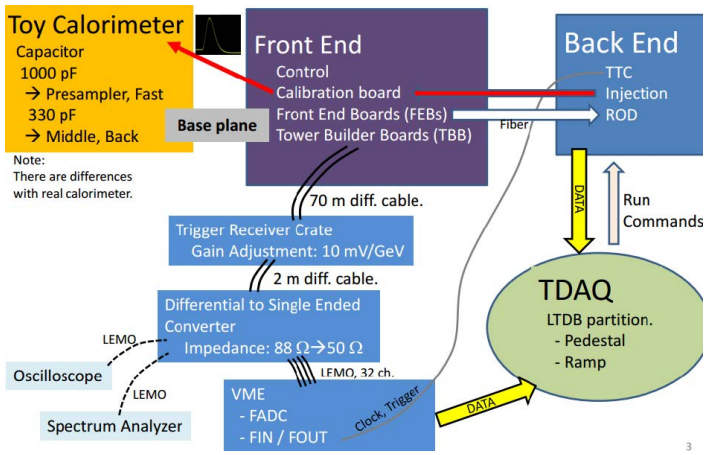

Fig. 6: The test system at LAr Electronic Maintenance Facility

Figure 6 shows the test system, a half front-end crate fully equipped with boards, and a readout system equivalent to the one operating in ATLAS are used in order to validate the 
new baseplane and the LTDB demonstrators. The calorimeter cells are simulated by a load which is plugged at the back of the baseplane. The calibration system can inject signals into the front end boards as if coming from the calorimeter. The output signal of the tower builder board is transmitted to the trigger receiver crate via a 70-meter cable. After a gain adjustment, this output signal is converted to single-ended, and then digitized by a Flash ADC. The output of the ADC is sent to the trigger and data acquisition system.

Different configurations have been tested, with the old or new baseplane and with or without LTDB. The LTDB has no obvious influence on the front-end boards outputs and the signals to analog trigger towers.

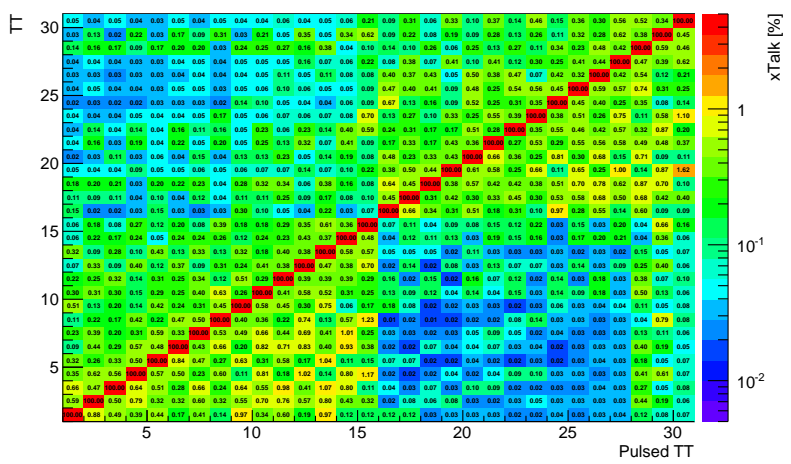

Fig. 7: Crosstalk between trigger towers

Figure 7 shows the crosstalk between trigger towers with one LTDB demonstrator on the new baseplane. When certain cells in all layers of one trigger tower are pulsed, the crosstalk to others trigger towers is smaller than $2 \%$.

\section{The Demonstrator in the Detector and Some Test Results}

After a review at CERN, the LTDB demonstrator has been approved by ATLAS. Both LTDB demonstrator boards have been installed into the crate on the detector. The blue fibers in Figure 8, one data link and one slow control link, are connected to the LTDB.

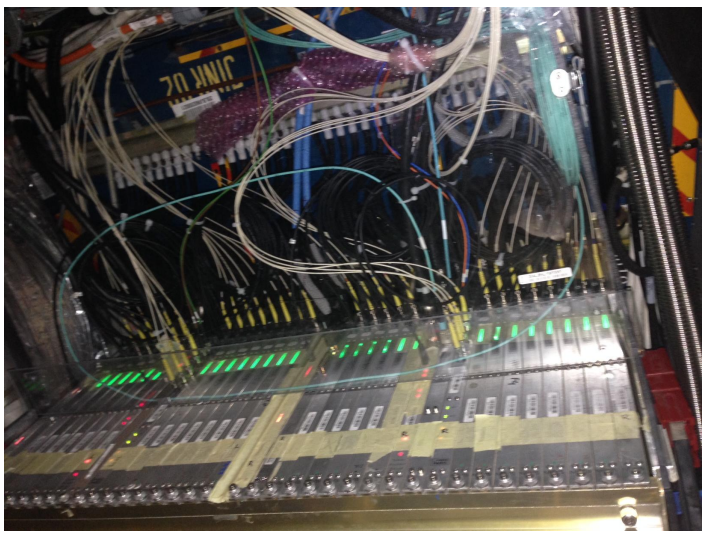

Fig. 8: The crate with LTDB demonstrators in the detector

A Gigabit Link Interface Board (GLIB) rack box is installed in the ATLAS counting room, and used for the LTDB slow control, and the FPGA reconfiguration. The ABBA board, and the PC controller are also in the same rack. The fibers from the LTDB board are connected to the GLIB rack box and the ABBA board.

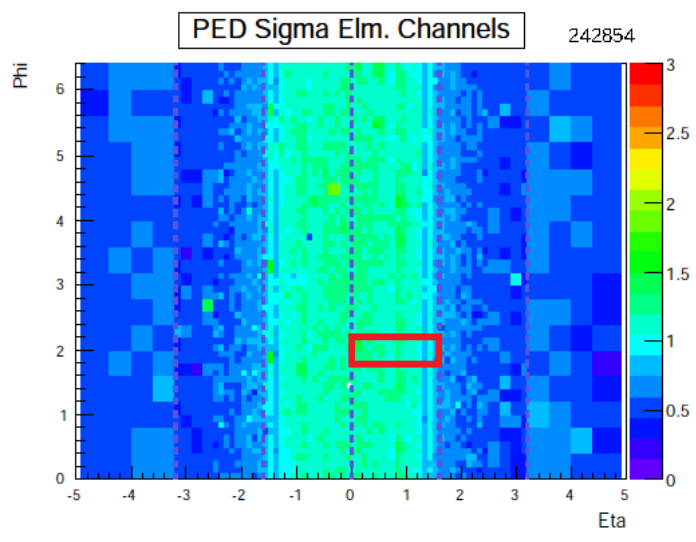

Fig. 9: Total noise of the LAr crates

The coverage of the crate with the installed demonstrator is ( $\eta=0-1.4, \phi=1.8-2.2)$, shown as the red block in Figure 9. This figure shows the total noise with all shaper switches on. The total noise level for this demonstrator crate is the same as that for the other crates in the same $\eta$ range.

\section{CONCLUSION}

The new LAr calorimeter trigger readout system is being designed for the Phase-I upgrade. The demonstrator system has been tested at LAr Electronic Maintenance Facility, before the installation. With satisfactory results obtained, both LTDB demonstrator boards have been installed on detector successfully. The preliminary results show that the total noise level of the crate with LTDB installed is at the same level of other crates. The LTDB demonstrators are being commissioned to operate in ATLAS for LHC Run 2, to study the performance of the new trigger readout electronics.

\section{REFERENCES}

[1] ATLAS Collaboration, The ATLAS Experiment at the CERN Large Hadron Collider, 2008 JINST 3 S08003

[2] ATLAS Collaboration, ATLAS Liquid Argon Calorimeter Technical Design Report, CERN-LHCC-96-041, http://cds.cern.ch/record/331061

[3] ATLAS Collaboration, ATLAS Liquid Argon Calorimeter PhaseI Upgrade Technical Design Report, CERN-LHCC-2013-017, http://cds.cern.ch/record/1602230 